\title{
Interaction-Induced Adiabatic Nonlinear Transport
}

\author{
Felix Reckermann, ${ }^{1,3}$ Janine Splettstoesser, ${ }^{2,3}$ and Maarten R. Wegewijs ${ }^{1,2,3}$ \\ ${ }^{1}$ Institut für Festkörper-Forschung-Theorie 3, Forschungszentrum Jülich, 52425 Jülich, Germany \\ ${ }^{2}$ Institut für Theoretische Physik A, RWTH Aachen University, 52056 Aachen, Germany \\ ${ }^{3}$ JARA-Fundamentals of Future Information Technology \\ (Received 16 April 2010; published 1 June 2010)
}

\begin{abstract}
We calculate the time-dependent nonlinear transport current through an interacting quantum dot in the single-electron tunneling (SET) regime. We show that an additional dc current is generated by the electron-electron interaction by adiabatic out-of-phase modulation of the gate and bias voltage. This current can arise only when two SET resonance conditions are simultaneously satisfied. We propose an adiabatic transport spectroscopy where lock-in measurement of a "time-averaged stability diagram" probes interactions, tunnel asymmetries, and changes in the ground state spin degeneracy.
\end{abstract}

DOI: 10.1103/PhysRevLett.104.226803

Introduction.-Transport through nanoscale devices modulated by time-dependent externally applied electric fields is an active field of research important for transport spectroscopy and manipulation of the charge and spin degrees of freedom in nanostructures; see, e.g., [1]. A particularly gentle way of time-dependently probing a system is through "adiabatic pumping" [2-4]. Here a finite dc current is generated in the absence of an applied bias by a weak, low frequency periodic modulation of system parameters. Adiabaticity in a transport situation means that many electrons visit the system during one cycle of the driving with frequency $\Omega$ and that the modulation is too weak and too slow to excite the device by direct state-tostate transitions. The current generated this way crucially depends on which subset of parameters is modulated, on the working point about which the modulation takes place, and on interactions, which are of special importance in nanoscale devices. For strictly adiabatic pumping one needs to vary at least two parameters, single-parameter pumping requiring a higher frequency [5]. Among the various combinations of parameters studied so far, the modulation of the applied bias has received little attention [6]. Most works have considered small deviations around an equilibrium working point where no steady state current is flowing. Adiabatic modulation around a nonequilibrium transport state induced by a static nonlinear bias voltage has been explored only for systems with negligible Coulomb interaction [4], motivated by experiments with surface acoustic waves [7]. Nonlinear bias voltage and Coulomb interaction have received little theoretical attention in the adiabatic regime. Limited to an equilibrium working point, some works have studied interacting quantum dots [8,9] and wires [10]. Including the effect of strong interactions beyond the mean-field picture is a challenge, since the powerful scattering matrix approach [2] breaks down here. Generally, one expects the additional nonequilibrium introduced by a static dc-bias voltage, in combination with strong electron-electron interactions, to strongly modify the pumping, providing novel opportuni-
PACS numbers: 73.23.Hk, 73.63.Kv, 85.35.-p

ties to investigate and control transport properties of nanoscale devices.

In this Letter we propose a new scheme for transport spectroscopy of interacting systems using adiabatically time-dependent electric fields. We analyze an interacting quantum dot in the single-electron tunneling (SET) regime, adiabatically driven by out-of-phase gate and bias potentials. In contrast to previous works, the applied bias can be arbitrary; i.e., we modulate the parameters around a steady nonequilibrium state supporting a finite dc current. We show that the strong local interaction generates an additional adiabatic de current, which is identically zero without interaction for any value of the applied voltages and magnetic field. We propose to use this effect as a tool for nonlinear transport spectroscopy which can be measured using lock-in techniques. The adiabatic dc current is nonzero only when two conditions for single-electron tunneling are simultaneously satisfied. Plotted as a function of the time-averaged gate and bias voltage, it gives rise to a new type of "stability diagram." Furthermore, we show that in an external magnetic field lifting the spin degeneracy, the adiabatic modulation only gives rise to transport effects in the regime of nonlinear bias, which qualitatively distinguish between different junction asymmetries.

Model.-We consider a quantum dot weakly coupled to two electrodes as sketched in Fig. 1(a). The gate and bias voltage are modulated with frequency $\Omega$ around the working point specified by the voltages $\bar{V}_{g}$ and $\bar{V}_{b}$ :

$$
V_{x}(t)=\bar{V}_{x}+\delta V_{x} \sin \left(\Omega t+\varphi_{x}\right), \quad x=b, g .
$$

We consider the important case where a single orbital level with strong Coulomb interaction $U$ is relevant for transport. We denote the spin-resolved dot number operator by $n_{\sigma}=d_{\sigma}^{\dagger} d_{\sigma}$, where the spin $\sigma=\uparrow, \downarrow$ is quantized along the external magnetic field (if present). The Hamiltonian reads $H_{D}(t)=\sum_{\sigma} \epsilon_{\sigma}(t) n_{\sigma}+U n_{\uparrow} n_{\downarrow}$. The energy of an electron created by $d_{\sigma}^{\dagger}$ equals $\epsilon_{\sigma}(t)=-\alpha V_{g}(t)+\sigma B / 2$ using the shorthand $\sigma= \pm 1$ for spin $\uparrow, \downarrow$. Importantly, the timedependent gate voltage $V_{g}(t)$ capacitively modulates this 

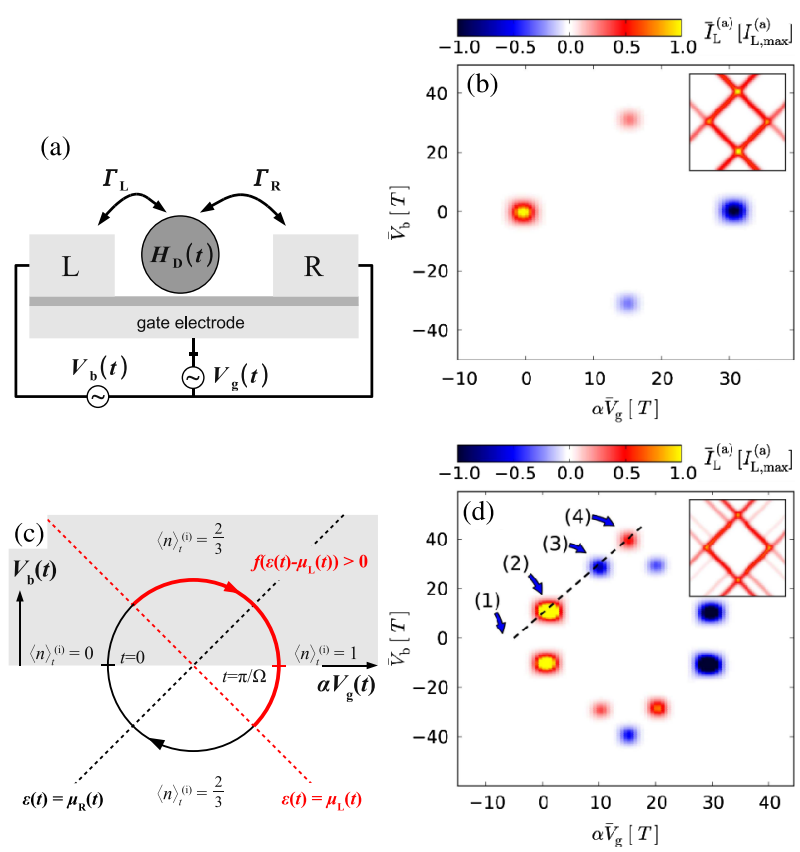

FIG. 1 (color online). (a) Sketch of the transport setup. (b) Adiabatic dc current through the left junction $\bar{I}_{L}^{(a)}$ as function of the time-averaged gate and bias voltage for Coulomb interaction $U=30 T$, zero magnetic field, and $\Gamma=0.5 T, \lambda=0.25$, and driving parameters $\Omega=0.1 T, \alpha \delta V_{g}=\delta V_{b}=0.5 T$. We plot $\bar{I}_{L}^{(a)}$ scaled to the maximal absolute value at the degeneracy points, $\bar{I}_{\text {max }, L}^{(a)}=\frac{2 \Omega}{27} \frac{\Gamma_{L} \Gamma_{R}}{(\Gamma / 2)^{2}} \frac{\alpha \delta V_{g}}{4 T} \frac{\delta V_{b}}{4 T}$, consisting of the frequency, a coupling asymmetry factor, and the ratio of the voltage phasespace factors to the thermal energy window. Inset: Differential conductance $d \bar{I}_{L}^{(i)} / d \bar{V}_{b}$ vs $\bar{V}_{g}$ and $\bar{V}_{b}$. (c) Voltage modulation cycle around the degeneracy point $\left(\alpha \bar{V}_{g}, \bar{V}_{b}\right) \approx(0,0)$ with dashed resonance lines $\varepsilon(t)=\mu_{r}(t)$. (d) Same as (b) for finite applied magnetic field, $B=10 T$.

energy with lever arm $\alpha<1$. Furthermore, $B$ is the Zeeman energy in units $e=\hbar=k_{B}=1$. The many-body eigenstates of $H_{D}(t)$ are $|0\rangle,|\sigma\rangle=d_{\sigma}^{\dagger}|0\rangle$ with $\sigma=\uparrow, \downarrow$, and $|2\rangle=d_{\uparrow}^{\dagger} d_{\downarrow}^{\dagger}|0\rangle$ with energies $0, \epsilon_{\sigma}(t), \sum_{\sigma} \epsilon_{\sigma}(t)+U$, respectively. The time-dependent bias $V_{b}(t)$ enters through the electrochemical potentials $\mu_{r}(t)= \pm V_{b}(t) / 2$ of electrodes $r=L, R$, which are described by $H_{E}(t)=$ $\sum_{r, k, \sigma}\left[\epsilon_{k}+\mu_{r}(t)\right] c_{r k \sigma}^{\dagger} c_{r k \sigma}$. Finally, $H_{T}=\sum_{r, k, \sigma} t_{r} c_{r k \sigma} d_{\sigma}^{\dagger}+$ H.c. describes the tunneling between the dot and the electrodes, with tunnel coupling strength $\Gamma_{r}=2 \pi \rho_{r}\left|t_{r}\right|^{2}$, where $t_{r}$ is the amplitude and $\rho_{r}$ the density of states of lead $r=L, R$. We define $\Gamma=\sum_{r} \Gamma_{r}$. We consider here the important case where the transport is affected dominantly through the modulation of the energy level positions $\varepsilon_{\sigma}(t)$ and the bias energy window $\mu_{L}(t)-\mu_{R}(t)$. The change in the tunnel coupling is of negligible importance. This is the typical situation in nonlinear transport spectroscopy of quantum dots. It holds, in particular, for small amplitude modulation of the voltages considered here.

Retarded occupations and transport current.-The total Hamiltonian $H(t)=H_{E}(t)+H_{D}(t)+H_{T}$ thus contains strong interaction on the dot, an adiabatic time dependence and nonequilibrium introduced by the nonlinear bias voltage. Within the framework of the real-time transport theory the time-dependent occupation probabilities of the manybody dot states, $\mathbf{p}(t)=\left(p_{0}(t), p_{\downarrow}(t), p_{\uparrow}(t), p_{2}(t)\right)$, can be shown to obey the kinetic equation [9]

$$
\dot{\mathbf{p}}(t)=\int_{-\infty}^{t} d t^{\prime} \mathbf{W}\left(t, t^{\prime}\right) \mathbf{p}\left(t^{\prime}\right) .
$$

The kernel, $\mathbf{W}\left(t, t^{\prime}\right)$, accounts for changes of the dot occupations due to electron tunnel processes to or from the electrodes. Although, it explicitly depends on both time arguments $t$ and $t^{\prime}$ (in contrast to the time-independent case), it can be calculated perturbatively for slowly varying fields [9]. Here we restrict ourselves to the lowest order contributions in both the tunneling coupling (SET) and in the time-dependent perturbation of external system parameters (adiabatic driving). We consistently solve the kinetic equation by expanding it around the instantaneous reference solution, $\mathbf{p}_{t}^{(i)}$, defined by

$$
\mathbf{0}=\mathbf{W}_{t}^{(i)} \mathbf{p}_{t}^{(i)}
$$

The instantaneous kernel and its zero-frequency Laplace transform $\quad \mathbf{W}_{t}^{(i)}=\lim _{\eta \downarrow 0} \int_{-\infty}^{t} d t^{\prime} \mathbf{W}^{(i)}\left(t-t^{\prime}\right) e^{\eta\left(t-t^{\prime}\right)}$ are evaluated using stationary transport theory in lowest order in $\Gamma$. In this limit of weak coupling, $\Gamma \ll T$, where $T$ is the electron temperature, the result reduces to Fermi's golden rule. In Eq. (3) the voltages are replaced by their instantaneous values at measuring time, $V_{x} \rightarrow V_{x}(t), x=b, g$, resulting in a parametric time dependence indicated by the subscript $t$. Hence, $\mathbf{p}_{t}^{(i)}$ would be the time-dependent steady state of the dot if the system was able to follow the parameter modulation instantaneously. By inserting $\mathbf{p}(t)=$ $\mathbf{p}_{t}^{(i)}+\mathbf{p}_{t}^{(a)}$ in Eq. (2), using Eq. (3), and doing a systematic lowest order expansion in $\Omega / \Gamma \ll 1$, we find for the first correction $\mathbf{p}_{t}^{(a)}$ to the instantaneous reference solution $\mathbf{p}_{t}^{(i)}$ :

$$
\dot{\mathbf{p}}_{t}^{(i)}=\mathbf{W}_{t}^{(i)} \mathbf{p}_{t}^{(a)}
$$

This correction accounts for the actual delay suffered by the system due to the finite rate of sweeping the voltages. Further corrections to this adiabatic approximation can be neglected if in addition $\alpha \delta V_{g}, \delta V_{b} \ll T^{2} / \Omega$. The timedependent steady state including the retardation is uniquely determined by Eqs. (3) and (4), together with the normalization conditions $\mathbf{e}^{T} \mathbf{p}_{t}^{(i)}=1$ and $\mathbf{e}^{T} \mathbf{p}_{t}^{(a)}=0$ with $\mathbf{e}^{T}=$ $(1,1,1,1)$.

The time-dependent current flowing from lead $r=L, R$ into the dot is found in a similar way [9] and can be decomposed into two corresponding parts, $I_{t, r}^{(i)}$ and $I_{t, r}^{(a)}$. Here $I_{t, r}^{(a)}$ is the adiabatic correction to the current due to the retardation of the system; i.e., it vanishes in the limit $\delta V \Omega \rightarrow 0$. The central quantities discussed in this Letter are obtained when averaging the two current 
contributions over one entire modulation cycle $\bar{I}_{r}^{(i \text { or } a)}=$ $\frac{\Omega}{2 \pi} \int_{0}^{2 \pi / \Omega} d t I_{t, r}^{(i \text { or } a)}$. Here, $\bar{I}_{r}^{(i)}$ equals the dc current one would measure for time-independent voltages equal to $\bar{V}_{g}$ and $\bar{V}_{b}$. Plotting $d \bar{I}_{L}^{(i)} / d \bar{V}_{b}$ as a function of these voltages, one obtains the standard Coulomb blockade stability diagram [1]. The quantity of central interest here, $\bar{I}_{r}^{(a)}$, is the additional dc current component due to the retardation of the quantum dot state. This quantity can be obtained, e.g., by subtracting from the total measured time-averaged current its zero-frequency limit.

For the time-dependent adiabatic current, we obtain a central analytic result:

$$
\begin{aligned}
I_{t, r}^{(a)}= & \frac{\left(\Gamma_{r}+\gamma_{r}\right)(\Gamma-\gamma)+\beta_{r} \beta}{\Gamma^{2}-\gamma^{2}+\beta^{2}} \frac{d}{d t}\langle n\rangle_{t}^{(i)} \\
& +2 \frac{\left(\Gamma_{r}+\gamma_{r}\right) \beta-\beta_{r}(\Gamma+\gamma)}{\Gamma^{2}-\gamma^{2}+\beta^{2}} \frac{d}{d t}\left\langle S_{z}\right\rangle_{t}^{(i)} .
\end{aligned}
$$

The average instantaneous charge, $\langle n\rangle_{t}^{(i)}=\sum_{\sigma} p_{t, \sigma}^{(i)}+$ $2 p_{t, 2}^{(i)}$, and spin, $\left\langle S_{z}\right\rangle_{t}^{(i)}=\sum_{t, \sigma}(\sigma / 2) p_{t, \sigma}^{(i)}$, are found from Eq. (3). Although the time-dependent adiabatic currents depend on the junction $r$ where they are evaluated, their time averages are related by charge conservation, $\sum_{r} I_{t, r}^{(a)}=$ $\frac{d}{d t}\langle n\rangle_{t}^{(i)}$, giving $\sum_{r} \bar{I}_{r}^{(a)}=0$. The prefactors in Eq. (5) contain

$$
\begin{gathered}
\gamma_{r}(t)=\frac{1}{2} \Gamma_{r} \sum_{\sigma}\left[f\left(\epsilon_{\sigma r}(t)\right)-f\left(\epsilon_{\sigma r}(t)+U\right)\right], \\
\beta_{r}(t)=\frac{1}{2} \Gamma_{r} \sum_{\sigma} \sigma\left[f\left(\epsilon_{\sigma r}(t)\right)-f\left(\epsilon_{\sigma r}(t)+U\right)\right],
\end{gathered}
$$

and their sums by $\gamma=\sum_{r} \gamma_{r}$, and $\beta=\sum_{r} \beta_{r}$, where $\sigma=$ \pm (corresponding to $\uparrow, \downarrow$ ) and $r=L, R$. All these quantities depend on time through the distance to resonance $\epsilon_{\sigma r}(t)=$ $\epsilon_{\sigma}(t)-\mu_{r}(t)$ in the arguments of the Fermi function $f(\omega)=[\exp (\omega / T)+1]^{-1}$. From Eq. (5) we infer a necessary condition for a nonvanishing time-averaged adiabatic current which also holds for more complex systems: $\bar{I}_{L}^{(a)}$ can only be nonzero if two SET resonance conditions are satisfied simultaneously. If only a single resonance condition is satisfied (effectively this is single-parameter pumping), (5) is a total time derivative of a periodic function, resulting in a zero time average. The resonances in $\bar{I}_{L}^{(a)}$ are thus located at resonance line crossings of the standard $d \bar{I}_{L}^{(i)} / d \bar{V}_{b}$ map.

Interaction-induced dc current.-The central result of this Letter relates to the prefactors in Eq. (5). Since the tunnel rates $\Gamma_{r}$ and $\Gamma=\sum_{r} \Gamma_{r}$ are time independent, it is clear that the adiabatic dc current is generated by the Coulomb interaction $U$. Indeed, since $\gamma_{r}=\beta_{r}=0$ for $U=0$ the adiabatic current $I_{t, r}^{(a)}=\left(\Gamma_{r} / \Gamma\right)\left(d\langle n\rangle_{t}^{(i)} / d t+\right.$ $\left.2 d\left\langle S_{z}\right\rangle_{t}^{(i)} / d t\right)$ is a total time derivative, which, integrated over a period, yields $\bar{I}_{r}^{(a)}=0$. We emphasize that in this case the current $\bar{I}_{r}^{(a)}$ vanishes identically for any value of the time-averaged external voltages and of the timeindependent tunnel couplings and external magnetic field. We now discuss the voltage dependence of the adiabatic dc current in the experimentally important regime of strong local interaction $U \gg T \gg \Gamma$. We fix the direction of the modulation cycle by taking $\varphi_{b}=0$ and $\varphi_{g}=-\pi / 2$, for which the adiabatic dc current is maximal, and time average the current numerically. We first focus on the case of zero magnetic field for which $\varepsilon_{\sigma}(t)=\varepsilon(t)=-\alpha V_{g}(t)$ is independent of spin $\sigma$. Therefore $\beta_{r}=0$ and Eq. (5) simplifies to $I_{t, r}^{(a)}=\left(\Gamma_{r}+\gamma_{r}\right) /(\Gamma+\gamma) d\langle n\rangle_{t}^{(i)} / d t$. In Fig. 1(b) we show a time-averaged stability diagram, i.e., $\bar{I}_{L}^{(a)}$ plotted as function of the time-averaged gate and bias voltage. In contrast to the standard $\left(d \bar{I}_{L}^{(i)} / d \bar{V}_{b}\right)$ stability diagram in the inset of Fig. 1(b), this map of pumped current indeed shows resonant enhancements only at discrete points of size $\propto T$ where two SET resonances meet. Most prominent are the two charge degeneracy points $\left(\alpha \bar{V}_{g}, \bar{V}_{b}\right) \approx(0,0)$ and $(U, 0)$ at which the adiabatic dc current has opposite sign and maximum amplitude. We now explain the microscopic origin of the positive sign of the adiabatic $\mathrm{dc}$ current at the degeneracy point $\left(\alpha \bar{V}_{g}, \bar{V}_{b}\right) \approx(0,0)$ for symmetric tunnel coupling $\Gamma_{L}=$ $\Gamma_{R}$. For $t \in(0, \pi / \Omega)$ the adiabatic current through the left junction is positive, $I_{t, L}^{(a)}>0$, whereas in the second half of the cycle $I_{t, L}^{(a)}<0$. This is because $\Gamma+\gamma \approx \Gamma[1+$ $\left.\sum_{r} f\left(\varepsilon(t)-\mu_{r}(t)\right) / 2\right]$ and $d\langle n\rangle_{t}^{(i)} / d t$ are symmetric and antisymmetric functions of the time $t$. The time average $\bar{I}_{L}^{(a)}$ is nevertheless nonzero due to the factor $\Gamma_{L}+\gamma_{L} \approx$ $\Gamma / 2+\Gamma f\left(\varepsilon(t)-\mu_{L}(t)\right) / 2$ in the numerator. Clearly, since the first term is constant, the nonzero time average comes from the contribution $\propto f\left(\varepsilon(t)-\mu_{L}(t)\right)$, which is nonzero for times for which $\varepsilon(t)<\mu_{L}(t)$ [gray (red) part of cycle in Fig. 1(c)]. One thus samples predominantly the loading parts of the cycle where $d\langle n\rangle_{t}^{(i)} / d t>0$ [shaded in Fig. 1(c)], where an excess of electrons tunnels onto the dot through the left junction. Therefore the adiabatic dc current is positive. Similarly, one finds for the point $\left(\alpha \bar{V}_{g}, \bar{V}_{b}\right) \approx(U, 0)$ the opposite adiabatic dc current due to the negative sign of the second term in Eq. (6). For asymmetric rates $\Gamma_{L} \neq \Gamma_{R}$ the time dependence of $\Gamma+\gamma$ becomes important as well, but does not alter the sign of the adiabatic dc current. In a magnetic field $B \gg T$ the adiabatic dc current plotted in Fig. 1(d) is completely suppressed in the linear response regime $\bar{V}_{b} \ll T$. Indeed, in this limit, $\gamma_{r}=\beta_{r}$, and $I_{t, r}^{(a)}$ has zero average [e.g., around $\alpha \bar{V}_{g} \ll T$ it is $\left.I_{t, r}^{(a)} \approx\left(\Gamma_{r} / \Gamma\right) d p_{t, \downarrow}^{(i)} / d t\right]$. In general, the interaction breaks the symmetry of loading and unloading parts of the cycle. The magnetic field, however, restores this symmetry in the linear response regime by lifting the spin degeneracy. Therefore, $\bar{I}_{r}^{(a)}$ is suppressed for $V_{b} \ll T$ even though $U \neq 0$. This is to be contrasted with the standard $d \bar{I}_{L}^{(i)} / d \bar{V}_{b}$ map shown in the inset, where 


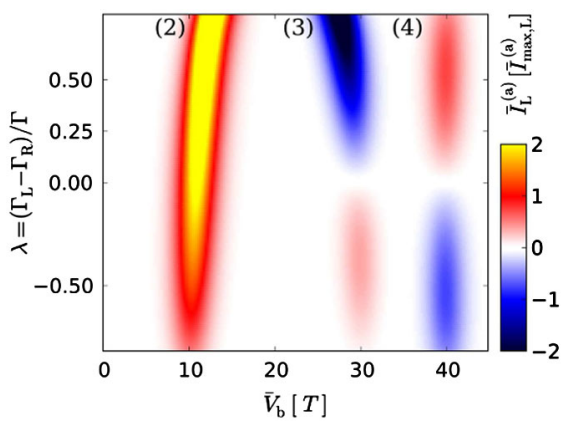

FIG. 2 (color online). The $\bar{I}_{L}^{(a)}$ along the line $\varepsilon=\mu_{R}$ [dashed line in Fig. 1(d)], as a function of the tunnel coupling asymmetry parameter $\lambda$, all other parameters being the same as in Fig. 1(d) and $\bar{I}_{\max , L}^{(a)}$ is taken for $\lambda=0\left(\Gamma_{L}=\Gamma_{R}\right)$.

in linear response the conductance shows the Coulomb oscillation peaks. Only at a finite voltage $\bar{V}_{b}=B$, where the spin-excited state becomes available, the loadingunloading symmetry is broken again and $\bar{I}_{r}^{(a)}$ is restored.

Coupling asymmetry.-Asymmetric rates induce additional features in Figs. 1(b) and 1(d) at finite bias $\left|\bar{V}_{b}\right|=U$ and $U-B$, respectively. In Fig. 2 we plot $\bar{I}_{L}^{(a)}$ along the dashed line in Fig. 1(a) as function of the coupling asymmetry, quantified by $\lambda=\left(\Gamma_{L}-\Gamma_{R}\right) / \Gamma$. Strikingly, the two high bias features marked (3) and (4) are qualitatively sensitive to the coupling asymmetry: if, e.g., resonance (3) is negative (positive), then $\Gamma_{L}>\Gamma_{R}\left(\Gamma_{L}<\Gamma_{R}\right)$. Quantitatively, for $\lambda>0$ the adiabatic dc current resonances marked (2) and (3) deviate from the "bare" resonance positions $(\lambda=0)$ by a shift which depends linearly on the temperature $T$ [11]. One can thus sensitively probe the coupling asymmetry.

Adiabatic spectroscopy.-Our results generalize to quantum dots with more complicated states and spectra: without interaction, the adiabatic dc current vanishes in leading order in $\Gamma$ and $\Omega$. Therefore, measurement of the time-averaged stability diagrams enables an adiabatic spectroscopy of nonlinear transport. Importantly, the occurrence of adiabatic dc current at sharply defined resonant points indicates that one is measuring in the adiabatic limit. This relates to the required effective two parameter modulation discussed with Eq. (5). Satisfying two SET resonance conditions simultaneously is, however, not yet sufficient for a nonzero average adiabatic current, as illustrated above for the crossing of the two ground-to-ground state resonances in a magnetic field. In general, the occurrence and sign of adiabatic dc current at a charge degeneracy point can be tied to the change in spin degeneracy in the ground state: the sign is positive (negative) if the ground state spin degeneracy increases (decreases) with the quantum dot charge and it vanishes if there is no change. The time-averaged stability diagram thus directly reveals nondegenerate ground states if $\bar{I}_{L}^{(a)}$ vanishes in the linear response regime. This may be interesting, e.g., for transport through magnetic molecules with high spin de- generacies or in carbon nanotubes where both spin and orbital degeneracies play a role. Another important aspect of the proposed spectroscopy is that the effects of "spurious" modulation of the barrier can be clearly identified experimentally. As shown in Ref. [9], a modulation of the gate voltage and of the barrier (instead of the bias voltage) results in an adiabatic de current which is symmetric with respect to reversal of the time-averaged gate voltage $\alpha \bar{V}_{g} \rightarrow U-\alpha \bar{V}_{g}$, in contrast to the antisymmetric shape found here. The proposed spectroscopy does furthermore not rely on quantum fluctuation effects and can therefore be observed readily in weakly coupled devices at moderate temperature and low driving frequency. We have checked that the corrections from next-to-leading order tunnel processes $\left(\Gamma^{2}\right)$ to the effects discussed here are quantitative and small, even for $\Gamma \sim T$. Importantly, even when including these corrections the adiabatic dc current still vanishes exactly for zero interaction. By measuring the proposed time-averaged stability diagram one thus gently probes junction asymmetries and strong interaction effects. This may prove valuable, for instance, in molecular quantum dots where stability is a key issue and transport is the only local probe available. Adiabatic transport through interacting nanosystems operated in the nonlinear regime is thus a promising topic where new experiments can be done.

We acknowledge discussion with S. Das, J. König, M. Plethyukhov, H. Schoeller, C. Stampfer, and financial support from the Helmholtz Foundation, the FZ-Jülich (IFMIT), and the Ministry of Innovation NRW.

[1] R. Hanson et al., Rev. Mod. Phys. 79, 1217 (2007).

[2] P. W. Brouwer, Phys. Rev. B 58, R10 135 (1998); M. Büttiker et al., Z. Phys. B 94, 133 (1994); M. Moskalets and M. Büttiker, Phys. Rev. B 66, 205320 (2002).

[3] F. Zhou et al., Phys. Rev. Lett. 82, 608 (1999); Y. Makhlin and A. D. Mirlin, ibid. 87, 276803 (2001); H. Pothier et al., Europhys. Lett. 17, 249 (1992); M. Switkes et al., Science 283, 1905 (1999).

[4] O. Entin-Wohlman et al., Phys. Rev. B 65, 195411 (2002).

[5] M. Grifoni and P. Hänggi, Phys. Rep. 304, 229 (1998).

[6] M. Moskalets and M. Büttiker, Phys. Rev. B 69, 205316 (2004).

[7] N.E. Fletcher et al., Phys. Rev. B 68, 245310 (2003); A. M. Robinson et al., ibid. 65, 045313 (2002).

[8] I. L. Aleiner and A. V. Andreev, Phys. Rev. Lett. 81, 1286 (1998); T. Aono, ibid. 93, 116601 (2004); E. Cota et al., ibid. 94, 107202 (2005); J. Splettstoesser et al., ibid. 95, 246803 (2005); E. Sela and Y. Oreg, ibid. 96, 166802 (2006); D. Fioretto and A. Silva, ibid. 100, 236803 (2008); L. Arrachea et al., Phys. Rev. B 77, 165326 (2008).

[9] J. Splettstoesser et al., Phys. Rev. B 74, 085305 (2006).

[10] R. Citro et al., Phys. Rev. B 68, 165312 (2003); S. Das and S. Rao, ibid. 71, 165333 (2005)

[11] M. M. Deshmukh et al., Phys. Rev. B 65, 073301 (2002); V. N. Golovach and D. Loss ibid. 69, 245327 (2004). 